\title{
Comparative study of various multiuser detection and base-station cooperation schemes for uplink multicell systems
}

\author{
Xiaojie Ju ${ }^{1 *}$, Lie-Liang Yang ${ }^{2}$ and Youguang Zhang ${ }^{1}$
}

\begin{abstract}
In this contribution, we investigate and compare the spectral-efficiency of uplink multicell systems, when various BS cooperation strategies and detection schemes are employed. Associated with our studies, three base-station (BS) operational schemes are considered, which are the ideal BS cooperation, data exchange only and no BS cooperation, in conjunction with four multiuser detection (MUD) schemes, including the optimum MUD (OMUD), OMUD with parallel interference cancellation (OMUD-PIC), linear minimum mean-square error MUD (MMSE-MUD) and the MMSE-based successive interference cancellation (MMSE-SIC). Their spectral-efficiency is evaluated either by simulations for the multicell systems of limited size or based on the formulas derived by asymptotic analysis. In this article, the asymptotic spectral-efficiency (ASE) of multicell systems with various BS cooperation and MUD schemes is derived based on the asymptotic analysis of the channel autocorrelation matrix's eigenvalue distribution or of the signal-to-interference-plus-noise ratio (SINR) attained by a concerned scheme. The impacts of system load, signal-to-noise ratio (SNR) and intercell interference strength on the spectral-efficiency are demonstrated. Our studies and performance results show that, when taking into account of the trade-off between complexity and performance, the MMSE-SIC supported by data exchange among BSs may constitute a promising multicell processing (MCP) scheme that is beneficial for implementation in practical systems.
\end{abstract}

Keywords: Multicell cooperation, Multiuser detection, Optimum multiuser detection, Minimum mean-square error, Interference Cancellation, Spectral-efficiency, Asymptotic spectral-efficiency

\section{Introduction}

With the increasing scarcity of spectrum, universal frequency reuse has been recognized as one of the important techniques in the future generations of cellular communication systems. In this case, intercell interference may become a dominant factor, which limits the spectralefficiency of cellular communication systems. In order to circumvent this problem, base-station (BS) cooperation has been introduced to suppress or even exploit intercell interference [1,2]. Usually, BS cooperation is achieved via exchanging some information, including channel state information (CSI) or/and user data, among the BSs involved with the aid of backhaul systems.

\footnotetext{
*Correspondence: juxiaojie798@hotmail.com

1 School of Electronic and Information Engineering, Beihang University, Beijing 100191, China

Full list of author information is available at the end of the article
}

The spectral-efficiency of cellular systems with/without BS cooperation has been widely investigated in literature, see [2] and the references therein, in order to quantify how efficient the spectrum resource is utilized and how much performance gain can be obtained by BS cooperation. Specifically, related to our studies in this contribution, in [3], the single-cell processing (SCP), which uses the detection based on the principles of minimum mean-square error and successive interference cancellation (MMSESIC), has been investigated in the context of the Wyner's infinite linear model [1]. The studies demonstrate that intercell interference has a dramatic effect on the achievable spectral-efficiency. Then, the studies in [3] have been extended to a more general model, which employs joint multicell processing (MCP) [4]. In [4], the infinite number of cells are assumed to be divided into clusters, where each cluster has $M$ cells and their $M$ BSs are assumed to cooperate ideally. In [5], the 'soft-handoff' scenario has been

\section{Springer}

(c) 2013 Ju et al.: licensee Springer. This is an Open Access article distributed under the terms of the Creative Commons Attribution License (http://creativecommons.org/licenses/by/2.0), which permits unrestricted use, distribution, and reproduction in any medium, provided the original work is properly cited. 
considered, where every mobile user simultaneously communicates with two BSs and is jointly controlled by the two BSs. The spectral-efficiency of both uplink and downlink has been analyzed under the assumption of ideal BS cooperation.

In the above-mentioned references, either no BS cooperation or ideal BS cooperation is assumed across the BSs involved. As the implementation of ideal BS cooperation usually requires a backhaul system with extremely high complexity, recently, BS cooperation supported by limited backhaul resources has been studied. Assuming a constrained backhaul system, in [6,7], the authors have investigated two BS cooperation schemes, namely, the distributed interference subtraction (DIS) and compressed interference forwarding (CIF), which only require to exchange the decoded user data or compressed user data among the involved BSs. However, in [6,7], each cell is assumed to support only one user. In [7], the capacity region has been studied, which is under the assumption of optimum multiuser detection (OMUD). The authors in [8] have considered the MCP based on the group MMSESIC, when assuming that each BS has multiple antennas but supports only one user. Most recently, a joint detection scheme has been investigated in [9], which turns an interference-limited system into a noise-limited system. Accordingly, intercell interference is exploited by acquiring the knowledge about the modulation formats of interfering users.

Against the background, in this article, we investigate and compare the achievable spectral-efficiency of the uplink multicell systems, which are modeled by the Wyner's infinite linear model [1], when various MUD and BS cooperation schemes are assumed. In contrast to the assumption of one user per cell that is usually used in the existing references, we assume that each BS employs multiple antennas and covers multiple users of each having a single transmit antenna, in order to study the impact of system load on the achievable spectral-efficiency of multicell systems. Associated with our studies, three levels of BS cooperation are considered, which are a) no BS cooperation, b) data exchange only and c) ideal BS cooperation, and four types of MUDs are addressed, which include a) optimum MUD (OMUD), b) OMUD with parallel intercell interference cancellation (OMUD-PIC), c) MMSEMUD and d) MMSE with successive intracell/intercell interference cancellation (MMSE-SIC). We first make use of the equivalent channel model to derive the formulas for the spectral-efficiency of multicell systems with various MUD and BS cooperation schemes. The requirements for carrying out BS cooperation are explained and the trade-off among the computational complexity, achievable spectral-efficiency and consumption of resources is discussed. Then, the asymptotic spectral-efficiency (ASE) of the multicell systems with fixed load-factors is analyzed with the aid of random matrix theory [10-14], also when the various MUD and BS cooperation schemes are considered. Furthermore, the special cases with the load-factor tending to zero, which are coincidence with the concept of massive MIMO [15-17], are analyzed. Finally, the spectral-efficiency performance of the multicell systems associated with the considered MUD and BS cooperation schemes is investigated via both the simulation results and the numerical results evaluated from the derived asymptotic formulas. Our studies and performance results show that, in general, using the OMUD with ideal BS cooperation is capable of attaining some extra spectral-efficiency against the other schemes considered, but at the cost of bandwidth and complexity for CSI exchange among BSs. In the heavily loaded multicell systems, the scheme of MMSE-SIC with data exchange stands above the other schemes that do not use CSI exchange. By contrast, when a multicell system is lightly loaded, making the load-factor approximately zero, which corresponds to the scenario of massive MIMO, all the schemes except the OMUD with ideal BS cooperation achieve a similar spectral-efficiency, which equals the spectral-efficiency achieved by an isolate cell with OMUD.

The rest of this article is structured as follows. Section 2 presents the system model. The spectral-efficiency of different schemes is derived in Section 3. In Section 4, we analyze the ASE, while in Section 5, the special cases with the load-factor tending to zero are considered. Performance results and discussions are provided in Section 6. Finally, in Section 7, we summarize the contributions and findings of this article.

\section{System model}

We consider the uplink transmission in a multicell SDMA system adhering to the Wyner's infinite linear model [1], as depicted in Figure 1. We assume that each BS employs $N$ receive antennas and supports $K$ uplink users of each having one transmit antenna. For the sake of simplicity to make the analysis manageable, the $K$ users in one cell are assumed to be co-located, so that they have a similar distance from the BS. As the typical assumptions used in the Wyner's model [1], we assume that intercell interference is only produced by two adjacent cells controlled by a common factor $0<\alpha \leq 1$, which becomes larger as the co-located users move close to the common edge of two adjacent cells. Depending on the specific multicell cooperation scheme considered, the BSs are assumed to be connected via ideal or limited backhaul links for data exchange and/or CSI sharing. The wireless channels from users to BSs are assumed to experience independent flat Rayleigh fading. Furthermore, power control is assumed, yielding that the total average power received from any intracell user is a constant, which is normalized to one, regardless of the number of receive antennas per BS. 


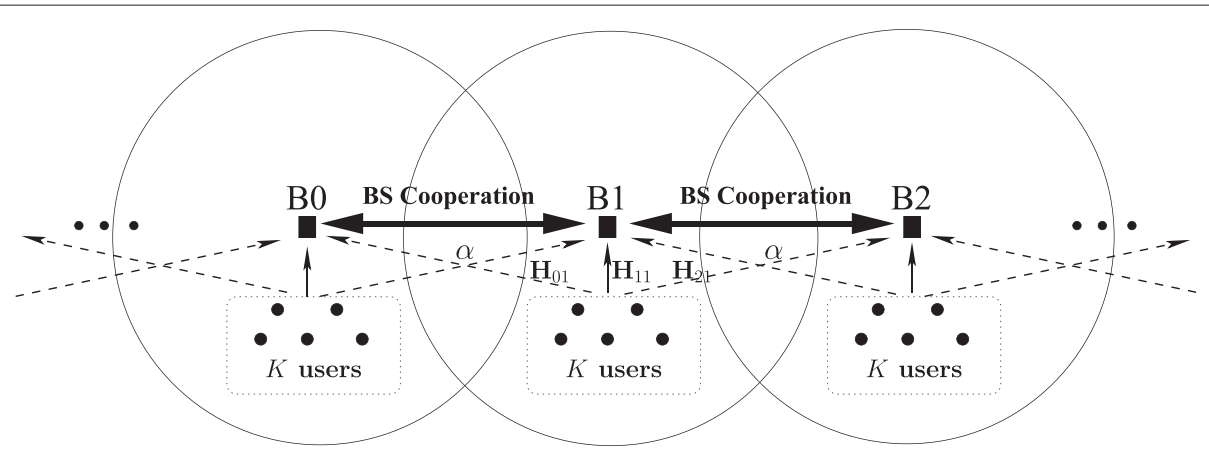

Figure 1 Extended Wyner's system model for multicell SDMA systems.

Based on the above assumptions, the MIMO equation for the observations of BS $i$ can be expressed as

$$
\begin{aligned}
\boldsymbol{y}_{i} & =\boldsymbol{H}_{i i} \boldsymbol{x}_{i}+\boldsymbol{H}_{i(i-1)} \boldsymbol{x}_{i-1}+\boldsymbol{H}_{i(i+1)} \boldsymbol{x}_{i+1}+\boldsymbol{n}_{i} \\
i & =\ldots,-1,0,1, \ldots
\end{aligned}
$$

where $\boldsymbol{y}_{i}$ is an $N$-length complex-valued observation vector, $\boldsymbol{x}_{i}=\left[x_{i 1}, x_{i 2}, \ldots, x_{i K}\right]^{T}$ contains the baseband symbols transmitted by the $K$ users controlled by BS $i$, while $\boldsymbol{H}_{i(i+j)}(j=-1,0,+1)$ is an $(N \times K)$ channel matrix, the elements of which obey the complex Gaussian distribution with zero mean and a common variance of $1 / N$ for $j=0$, which corresponds to the intracell users, or $\alpha^{2} / N$ for $j=-1,+1$, which corresponds to the users in the two adjacent cells. We assume that the CSI is only available to the receivers at the BSs, while the mobile users only make use of the channel distribution information (CDI) for signal transmission. In (1), $\boldsymbol{n}_{i}$ is an $N$-length complex-valued noise vector, which obeys the complex Gaussian distribution with zero mean and a covariance matrix of $\sigma^{2} \boldsymbol{I}$ with $\sigma^{2}$ the noise variance.

Based on the above system model, let us now consider the spectral-efficiency of the uplink multicell SDMA systems, when various BS cooperation and multiuser detection schemes are assumed.

\section{Spectral-efficiency}

In this section, the spectral-efficiency of uplink multicell SDMA systems is investigated, when various multicell cooperation and detection strategies are considered. The spectral-efficiency is expressed in terms of bits per second per hertz per user(bits/s/Hz/User). Let us first consider the OMUD with ideal BS cooperation.

\subsection{Optimum multiuser detection with ideal BS cooperation}

In the context of this scenario, we assume that the BSs detect their signals jointly by the OMUD, which is supported by ideal sharing of both the CSI and the received observations of all the cells. In other words, under the ideal BS cooperation, it seems that there is a visual signal processing center, which employs the ideal CSI as well as the observations obtained by all the BSs involved. As shown in Figure 1, although each cell is only related to two adjacent cells, all the cells are dependent on each other. Hence, when the OMUD with ideal BS cooperation is considered, we have to consider all the cells involved. For this sake, let

$$
\begin{aligned}
& \boldsymbol{y}=\left[\ldots, \boldsymbol{y}_{-1}^{T}, \boldsymbol{y}_{0}^{T}, \boldsymbol{y}_{1}^{T}, \ldots\right]^{T} \\
& \boldsymbol{x}=\left[\ldots, \boldsymbol{x}_{-1}^{T}, \boldsymbol{x}_{0}^{T}, \boldsymbol{x}_{1}^{T}, \ldots\right]^{T} \\
& \boldsymbol{n}=\left[\ldots, \boldsymbol{n}_{-1}^{T}, \boldsymbol{n}_{0}^{T}, \boldsymbol{n}_{1}^{T}, \ldots\right]^{T}
\end{aligned}
$$

Then, $\boldsymbol{y}$ can be expressed as

$$
\boldsymbol{y}=\boldsymbol{H} \boldsymbol{x}+\boldsymbol{n}
$$

where the channel matrix has infinite dimensions and is in the form of

$$
\boldsymbol{H}=\left[\begin{array}{cccccccc}
\cdots & \ddots & \ddots & \ddots & \ldots & & & \\
& \cdots & \boldsymbol{H}_{0(-1)} & \boldsymbol{H}_{00} & \boldsymbol{H}_{01} & \ldots & & \\
& \cdots & \boldsymbol{H}_{10} & \boldsymbol{H}_{11} & \boldsymbol{H}_{12} & \ldots & \\
& & \cdots & \boldsymbol{H}_{21} & \boldsymbol{H}_{22} & \boldsymbol{H}_{23} & \ldots & \\
& & & \ldots & \ddots & \ddots & \ddots & \ldots
\end{array}\right]
$$

where except the terms expressed as $\boldsymbol{H}_{i j}$ with $j=i-1$, $i, i+1$, the other entries are zero matrices. From (3) the spectral-efficiency of the multicell SDMA systems with OMUD and ideal BS cooperation can be expressed as [2]

$$
C=\lim _{M \rightarrow \infty} \frac{1}{M K} E\left[\log _{2} \operatorname{det}\left(\boldsymbol{I}+\frac{1}{\sigma^{2}} \boldsymbol{H} \boldsymbol{H}^{H}\right)\right]
$$

where $E[\cdot]$ denotes the expectation with respect to the channel matrix, while $M \rightarrow \infty$ indicates that an infinite number of BSs are invoked. 
The spectral-efficiency evaluated by (5) for the model (3) is the spectral-efficiency of the multicell system with an infinite number of BSs arranged on a line. As shown in [4], this spectral-efficiency is equal to that of the multicell system with $M \geq 3$ number of BSs arranged evenly on a circle. Furthermore, it has been demonstrated in [4] that the spectral-efficiency of the multicell systems with circularly distributed BSs is independent of the value of $M$, provided that $M \geq 3$. In this case, the spectral-efficiency of (5) can be evaluated from the multicell system with three BSs arranged on a circle with an equal distance from each other, as shown in Figure 2, and can be expressed as

$$
C=\frac{1}{3 K} E\left[\log _{2} \operatorname{det}\left(\boldsymbol{I}+\frac{1}{\sigma^{2}} \boldsymbol{H} \boldsymbol{H}^{H}\right)\right],
$$

associated with the channel matrix expressed as

$$
\boldsymbol{H}=\left[\begin{array}{lll}
\boldsymbol{H}_{00} & \boldsymbol{H}_{01} & \boldsymbol{H}_{02} \\
\boldsymbol{H}_{10} & \boldsymbol{H}_{11} & \boldsymbol{H}_{12} \\
\boldsymbol{H}_{20} & \boldsymbol{H}_{21} & \boldsymbol{H}_{22}
\end{array}\right]
$$

Since the OMUD with ideal BS cooperation is considered, explicitly, the spectral-efficiency evaluated from (6) is an upper bound for all the other BS cooperation schemes associated with various BS detection schemes. The ideal BS cooperation exploits the intercell interference positively rather than eliminates it. However, the complexity for implementation of ideal BS cooperation, especially, with OMUD is extreme. Furthermore, exchanging both the CSI and the observations of many invoked BSs requires a backhaul system having huge bandwidth and, possibly, spending a lot of energy. In the following sections, more practical BS cooperation and detection schemes are considered, which usually have significantly lower complexity than the OMUD with ideal BS cooperation. Furthermore, the bandwidth and energy required for information exchange among $\mathrm{BSs}$ by the backhaul system can also be significantly reduced.

\subsection{Optimum multiuser detection with ideal data exchange}

To reduce the bandwidth and energy required by the backhaul system for implementing ideal BS cooperation, BSs may refrain from sharing CSI, but only exchange their data received from mobile users. In this case, when the OMUD is employed, PIC can be carried out after a BS obtains the data detected by the other BSs. This scheme is referred to as the OMUD-PIC, which is implemented as follows.

Let us first assume that the extended Wyner's linear model shown in Figure 1 includes $M=2 m+1$ cells indexed from left to right as $-m,-m+1, \ldots, m$. When the OMUD-PIC is employed, BS $(-m)$ carries out detection under the interference from Cell $(-m+1)$. After the detection of BS $(-m)$, it sends the detected data to BS $(-m+1)$. Then, BS $(-m+1)$ detects its $K$ users after canceling the interference from Cell $(-m)$, as it employs the CSI of the channels from the $K$ users in Cell $(-m)$ to BS $(-m+1)$. The above procedure continues in sequence until BS $m$ detects its $K$ users. From the above description, we know that, except BS $m$, every BS detects its $K$ users in the presence of the intercell interference from one

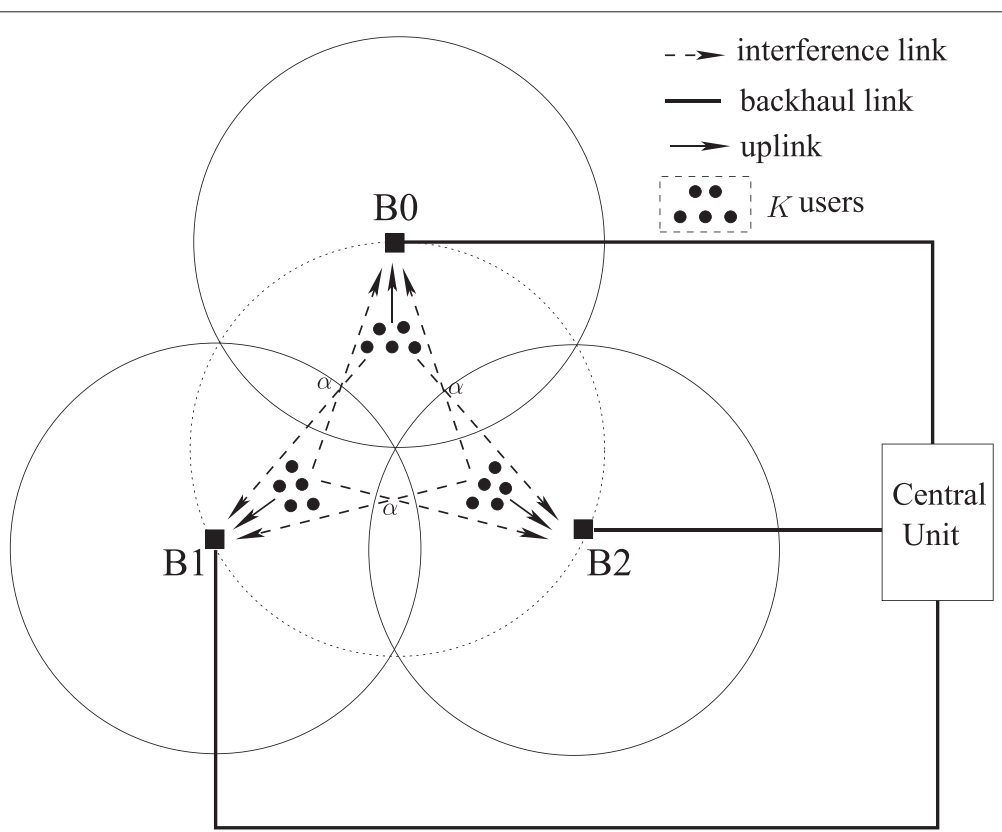

Figure 2 Circle Wyner's system model with three cooperative BSs. 
cell at its right. The rightmost BS $m$ does not experience any intercell interference after canceling the interference from Cell $(m-1)$. Therefore, based on [18], the spectralefficiency per user for all the cells except Cell $m$ can be expressed as

$$
\begin{aligned}
C_{i} & =\frac{1}{K} E\left[\log _{2} \operatorname{det}\left(\boldsymbol{I}+\boldsymbol{H}_{i i} \boldsymbol{H}_{i i}^{H} \boldsymbol{\Sigma}_{i(i+1)}^{-1}\right)\right], \\
i & =-m,-m+1, \ldots, m-1,
\end{aligned}
$$

where $\boldsymbol{\Sigma}_{i(i+1)}=\boldsymbol{H}_{i(i+1)} \boldsymbol{H}_{i(i+1)}^{H}+\sigma^{2} \boldsymbol{I}$ denotes the covariance matrix of the interference imposed by Cell $(i+1)$ on Cell $i$ plus Gaussian noise. Considering the symmetric structure of the Wyner's linear model, the above spectralefficiency is independent of the index $i$. Hence, in our forthcoming discourses, we consider only Cell 1 and its two neighbors, Cell 0 and 2, as well as the corresponding overall channel matrix given by (7), unless specifically notified. By contrast, the spectral-efficiency per user for Cell $m$ is the same as that of an isolate cell, given by [19]

$$
C_{m}=\frac{1}{K} E\left[\log _{2} \operatorname{det}\left(\boldsymbol{I}+\frac{1}{\sigma^{2}} \boldsymbol{H}_{m m} \boldsymbol{H}_{m m}^{H}\right)\right] .
$$

Consequently, the average spectral-efficiency achieved by the OMUD-PIC in the context of the Wyner's infinite linear model is

$$
C=\lim _{M \rightarrow \infty} \frac{1}{M} \sum_{i} C_{i}=\frac{1}{K} E\left[\log _{2} \operatorname{det}\left(\boldsymbol{I}+\boldsymbol{H}_{11} \boldsymbol{H}_{11}^{H} \Sigma_{12}^{-1}\right)\right] .
$$

It can be seen that, under the OMUD-PIC, one cell only needs to send its detected data to one of its two neighbors. Hence, in comparison with the ideal BS cooperation, as discussed in Section 3.1, the requirements imposing on the backhaul system can be significantly relaxed. However, the OMUD, such as, the maximum likelihood detector [20], is still very high-complexity, which usually becomes extreme when the number of users supported per cell is relatively high. Therefore, we below consider a range of suboptimum MUD schemes that are more practical.

\subsection{MMSE-MUD without BS cooperation}

In order to illustrate the performance enhancement by BS cooperation, we first give the spectral-efficiency of two related MUD benchmark schemes without employing BS cooperation. The first one is the MMSE-MUD, which is discussed in this section, and the other one is the MMSE-SIC, which is addressed in Section 3.4.

When the MMSE-MUD is employed by Cell 1, which uses no information from the other cells, it is well known that the SINR of detection can be expressed as [21]

$$
\gamma_{1}=\boldsymbol{h}_{11,1}^{H} \boldsymbol{R}_{I}^{-1} \boldsymbol{h}_{11,1}
$$

where

$$
\boldsymbol{R}_{I}=\left(\boldsymbol{H}_{11} \boldsymbol{H}_{11}^{H}+\boldsymbol{\Sigma}-\boldsymbol{h}_{11,1} \boldsymbol{h}_{11,1}^{H}\right)
$$

represents the covariance matrix of the interference (both intracell and intercell) plus noise, $\boldsymbol{\Sigma}=\boldsymbol{H}_{10} \boldsymbol{H}_{10}^{H}+$ $\boldsymbol{H}_{12} \boldsymbol{H}_{12}^{H}+\sigma^{2} \boldsymbol{I}$ represents the covariance matrix of the overall intercell interference plus noise, while $\boldsymbol{h}_{11,1}$ is the first column of $\boldsymbol{H}_{11}$, as seen in (7).

Consequently, when the multicell systems employ no BS cooperation and, at each BS, the MMSE-MUD is employed, the spectral-efficiency per user can be evaluated from the formula

$$
C=E\left[\log _{2}\left(1+\gamma_{1}\right)\right]
$$

where $E[\cdot]$ takes the expectation with respect to $\gamma_{1}$.

\subsection{MMSE-SIC without BS cooperation}

It is well-known that the SIC assisted MUDs (SIC-MUDs) constitute a class of detectors, which are capable of achieving the sum capacity [22] and, in principle, approximate the ML-MUD [20]. Among the SIC-MUDs, the MMSESIC is the one that has been widely studied. It can be shown that, when the system size is relatively large, the MMSE-SIC is capable of achieving the near optimum error performance, even when symbol-by-symbol detection is considered [23]. In this section, we first illustrate how the MMSE-SIC achieves the sum capacity. Then, some discussion about the detection procedure is provided.

Since no BS cooperation is assumed, the MMSE-SIC of a BS detects the $K$ user signals using $K$ stages, detecting one at each stage. Specifically for Cell 1 , in the first stage, the first user is detected in the same way as the MMSE-MUD considered in Section 3.3, yielding the SINR as shown in (11). Hence, the spectral-efficiency of user 1 , which is expressed as $C_{1}$, is given by (13).

After the detection of the first user, its interference on the other $(K-1)$ users is then canceled by forming a new observation vector

$$
\boldsymbol{y}^{(1)}=\boldsymbol{y}-\boldsymbol{h}_{11,1} x_{11}
$$

where the interference cancellation is ideal, as the error probability of user 1 is zero, provided that its information rate does not exceed $C_{1}$. In the following stages, the other users are detected in the same way as user 1 ; one user is detected at every stage and, then, its interference on those having not been detected is canceled. This process is repeated until all the $K$ users are detected. 
Let us define

$$
\begin{aligned}
\boldsymbol{R}_{I}^{(0)} & =\boldsymbol{H}_{11} \boldsymbol{H}_{11}^{H}+\boldsymbol{\Sigma} \\
\boldsymbol{R}_{I}^{(k)} & =\boldsymbol{H}_{11} \boldsymbol{H}_{11}^{H}+\boldsymbol{\Sigma}-\sum_{i=1}^{k} \boldsymbol{h}_{11, i} \boldsymbol{h}_{11, i}^{H} \\
& =\boldsymbol{R}_{I}^{(0)}-\sum_{i=1}^{k} \boldsymbol{h}_{11, i} \boldsymbol{h}_{11, i}^{H} \\
& =\boldsymbol{R}_{I}^{(k-1)}-\boldsymbol{h}_{11, k} \boldsymbol{h}_{11, k}^{H}, k=1,2, \ldots, K
\end{aligned}
$$

Then, according to the principles of the MMSE-SIC, after completing the detection of $(k-1)$ users and canceling their interference on the other users, the SINR for detection of the $k$ th user at the $k$ th detection stage is given by

$$
\gamma_{k}=\boldsymbol{h}_{11, k}\left(\boldsymbol{R}_{I}^{(k)}\right)^{-1} \boldsymbol{h}_{11, k}^{H}, k=1,2, \ldots, K
$$

Correspondingly, the spectral-efficiency of user $k$ is

$$
\begin{aligned}
C_{k} & =E\left[\log _{2}\left(1+\gamma_{k}\right)\right] \\
& =E\left[\log _{2}\left(1+\boldsymbol{h}_{11, k}\left(\boldsymbol{R}_{I}^{(k)}\right)^{-1} \boldsymbol{h}_{11, k}^{H}\right], k=1,2, \ldots, K\right.
\end{aligned}
$$

From (15), we have

$$
\begin{aligned}
\boldsymbol{R}_{I}^{(k-1)} & =\boldsymbol{R}_{I}^{(k)}+\boldsymbol{h}_{11, k} \boldsymbol{h}_{11, k}^{H} \\
& =\boldsymbol{R}_{I}^{(k)}\left[\boldsymbol{I}_{N}+\left(\boldsymbol{R}_{I}^{(k)}\right)^{-1} \boldsymbol{h}_{11, k} \boldsymbol{h}_{11, k}^{H}\right]
\end{aligned}
$$

Upon taking the determinant on both sides of (18), we can arrive at

$$
\begin{aligned}
\operatorname{det}\left(\boldsymbol{R}_{I}^{(k-1)}\right) & =\operatorname{det}\left(\boldsymbol{R}_{I}^{(k)}\right) \times \operatorname{det}\left[\boldsymbol{I}_{N}+\left(\boldsymbol{R}_{I}^{(k)}\right)^{-1} \boldsymbol{h}_{11, k} \boldsymbol{h}_{11, k}^{H}\right] \\
& =\operatorname{det}\left(\boldsymbol{R}_{I}^{(k)}\right) \times\left[1+\boldsymbol{h}_{11, k}^{H}\left(\boldsymbol{R}_{I}^{(k)}\right)^{-1} \boldsymbol{h}_{11, k}\right]
\end{aligned}
$$

When using this relationship into (17), the spectralefficiency of user $k$ can be written as

$$
\begin{aligned}
C_{k} & =E\left[\log _{2} \operatorname{det}\left(\boldsymbol{R}_{I}^{(k-1)}\right)\right]-E\left[\log _{2} \operatorname{det}\left(\boldsymbol{R}_{I}^{(k)}\right)\right], \\
k & =1,2, \ldots, K
\end{aligned}
$$

The average spectral-efficiency per user over the $K$ users of Cell 1 is

$$
\begin{aligned}
C & =\frac{1}{K} \sum_{k=1}^{K} C_{k} \\
& =\frac{1}{K} \sum_{k=1}^{K}\left\{E\left[\log _{2} \operatorname{det}\left(\boldsymbol{R}_{I}^{(k-1)}\right)\right]-E\left[\log _{2} \operatorname{det}\left(\boldsymbol{R}_{I}^{(k)}\right)\right]\right\} \\
& =\frac{1}{K}\left\{E\left[\log _{2} \operatorname{det}\left(\boldsymbol{R}_{I}^{(0)}\right)\right]-E\left[\log _{2} \operatorname{det}\left(\boldsymbol{R}_{I}^{(K)}\right)\right]\right\} \\
& =\frac{1}{K} E\left[\log _{2} \operatorname{det}\left(\boldsymbol{H}_{11} \boldsymbol{H}_{11}^{H}+\boldsymbol{\Sigma}\right)-\log _{2} \operatorname{det}(\boldsymbol{\Sigma})\right] \\
& =\frac{1}{K} E\left[\log _{2} \operatorname{det}\left(\boldsymbol{I}+\boldsymbol{H}_{11} \boldsymbol{H}_{11}^{H} \boldsymbol{\Sigma}^{-1}\right)\right] .
\end{aligned}
$$

It can be readily observed that (21) has the same form as (8), which is the spectral-efficiency of the optimum detector analyzed in [18] when there exists interference. This explains that the MMSE-SIC without BS cooperation is capable of achieving the same capacity as the optimum MUD without BS cooperation. Note that, the reason behind this conclusion is that, according to ([24], 8.3.4), the MMSE processing is information-lossless. Hence, the spectral-efficiency achieved at each stage is precisely the maximum mutual information between the detected symbol and the received signal. Consequently, the total spectral-efficiency is just the channel capacity.

In order to achieve the spectral-efficiency given by (21), the BS of a cell requires to inform its $K$ mobile users at which rates they should transmit. A user detected at an earlier stage must transmit at a lower rate than a user detected at a later stage, as the SINR of a later detected user is higher than that of an earlier detected one, owing to the interference cancellation. This detection process explicitly results in unfairness. In order to enhance the fairness, the detection order may be updated periodically. However, in this case, extra resource is required to inform the mobile users the change of ordering. Moreover, joint coding that considers different data rates is required by each user.

In order to make the communication fair for all the mobile users, alternatively, every mobile user may transmit at the same rate, such as at $C$ given in (21). According to [23], channel reliability knowledge can be exploited by the receiver to improve the error performance. Specifically, at the BS receiver, the detection is carried out in the order from the more reliable ones to the less reliable ones. By doing this, the users detected at earlier stages benefit from the high channel reliability, making their channel capacities higher than their transmission rates. Hence, they can be reliably detected. By contrast, the later detected users can benefit from the interference 
cancellation operations. Owing to the interference cancellation, the SINR of later detected users improves, which in turn results in improved channel capacities. Hence, the detection reliabilities of the later detected users will also improve. This in fact explains why in [23], when multiuser diversity is exploited for detection, the MMSE-SIC is capable of achieving the near optimum error performance, especially in the cases when the system is relatively large.

\subsection{MMSE-SIC with ideal data exchange}

The MMSE-SIC without BS cooperation is capable of achieving the spectral-efficiency of the optimum MUD without BS cooperation. However, the intercell interference significantly degrades the achievable spectralefficiency. With the aid of BS cooperation by exchanging the data detected by adjacent BSs at different detection stages, the spectral-efficiency of multicell systems employing the MMSE-SIC can be significantly increased. Below we consider this scenario.

When operated under the scheme of MMSE-SIC with ideal data exchange, multiple stages of detection in the principles of the MMSE-SIC are exploited, so that the data detected at a stage can be shared by the BSs, in order to cancel their interference on the following stages of detection. To be more specific, under the MMSE-SIC with ideal data exchange, at every stage of detection, each of the three BSs detects one user. Then, the detected symbol is sent via the backhaul-links to the other two BSs. Simultaneously, it also receives the two symbols detected by the other BSs. In the next stage of detection, the interference imposed by these three symbols is canceled. The above process is continued until all the users in each cell are detected.

Let us assume that the detection process is at the $k$ th stage for detection of user $k$. In this case, owing to the data exchange between adjacent BSs, the covariance matrix of interference plus noise at BS 1 can be written as

$$
\boldsymbol{R}_{I}^{(k)}=\left(\boldsymbol{H}_{11} \boldsymbol{H}_{11}^{H}+\boldsymbol{\Sigma}-\sum_{i=1}^{k} \boldsymbol{h}_{11, i} \boldsymbol{h}_{11, i}^{H}-\sum_{j=0}^{k-1} \boldsymbol{\Psi}_{j}\right),
$$

where, by definition, $\boldsymbol{\Psi}_{0}=\mathbf{0}, \boldsymbol{\Psi}_{j}=\left(\boldsymbol{h}_{10, j} \boldsymbol{h}_{10, j}^{H}+\right.$ $\left.\boldsymbol{h}_{12, j} \boldsymbol{h}_{12, j}^{H}\right)$, which is contributed by the data exchange occurred after the $j$ th detection stage. Hence, when the MMSE-SIC scheme is employed, the SINR at the $k$ th detection stage is $\gamma_{k}=\boldsymbol{h}_{11, k}^{H}\left(\boldsymbol{R}_{I}^{(k)}\right)^{-1} \boldsymbol{h}_{11, k}$ and, correspondingly, the spectral-efficiency of user $k$ of Cell 1 is given by [21]

$$
C_{k}=E\left[\log _{2}\left(1+\boldsymbol{h}_{11, k}^{H}\left(\boldsymbol{R}_{I}^{(k)}\right)^{-1} \boldsymbol{h}_{11, k}\right)\right], k=1,2, \ldots, K
$$

Furthermore, the average spectral-efficiency per user of the multicell systems using the MMSE-SIC with ideal data exchange is

$$
C=\frac{1}{K} \sum_{k=1}^{K} C_{k}
$$

The spectral-efficiency of (23) can also be expressed in other forms. As an example, the covariance matrix $\boldsymbol{R}_{I}^{(k)}$ in (22) can be expressed in a recursive form as

$$
\boldsymbol{R}_{I}^{(k)}=\boldsymbol{R}_{I}^{(k-1)}-\boldsymbol{h}_{11, k} \boldsymbol{h}_{11, k}^{H}-\boldsymbol{\Psi}_{k-1}
$$

After some arrangement, this equation can be written as

$$
\boldsymbol{R}_{I}^{(k)}\left(\boldsymbol{I}_{N}+\left(\boldsymbol{R}_{I}^{(k)}\right)^{-1} \boldsymbol{h}_{11, k} \boldsymbol{h}_{11, k}^{H}\right)=\boldsymbol{R}_{I}^{(k-1)}-\boldsymbol{\Psi}_{k-1}
$$

Hence, by following the approaches used in Section 3.4, we can obtain

$$
\begin{aligned}
& \log _{2}\left(1+\boldsymbol{h}_{11, k}^{H}\left(\boldsymbol{R}_{I}^{(k)}\right)^{-1} \boldsymbol{h}_{11, k}\right) \\
= & \log _{2} \operatorname{det}\left(\boldsymbol{R}_{I}^{(k-1)}-\boldsymbol{\Psi}_{k-1}\right)-\log _{2} \operatorname{det}\left(\boldsymbol{R}_{I}^{(k)}\right) \\
= & \log _{2} \operatorname{det}\left[\left(\boldsymbol{R}_{I}^{(k-1)}-\boldsymbol{\Psi}_{k-1}\right)\left(\boldsymbol{R}_{I}^{(k)}\right)^{-1}\right]
\end{aligned}
$$

Consequently, $C_{k}$ in (23) can be written as

$$
\begin{aligned}
C_{k} & =E\left[\log _{2} \operatorname{det}\left(\boldsymbol{R}_{I}^{(k-1)}-\boldsymbol{\Psi}_{k-1}\right)\right]-E\left[\log _{2} \operatorname{det}\left(\boldsymbol{R}_{I}^{(k)}\right)\right] \\
& =E\left[\log _{2} \operatorname{det}\left[\left(\boldsymbol{R}_{I}^{(k-1)}-\boldsymbol{\Psi}_{k-1}\right)\left(\boldsymbol{R}_{I}^{(k)}\right)^{-1}\right]\right], \\
k & =1,2, \ldots, K
\end{aligned}
$$

Let us now analyze the asymptotic spectral-efficiency of the BS cooperation and detection schemes considered in this contribution.

\section{Analysis of asymptotic spectral-efficiency}

In this section, we analyze the asymptotic spectralefficiency (ASE) of the MUD and BS cooperation schemes considered in Section 3 based on the random matrix theory, when assuming that $K, N \rightarrow \infty$ while $\beta=K / N<\infty$ is fixed, where $\beta$ is referred to as the system load-factor. As done in literature $[3,11,25]$, the ASE is analyzed by deriving either the asymptotic eigenvalue distribution of the matrices in the form of $\boldsymbol{H H}^{H}$ [11] or the asymptotic distribution of the SINR attained by the various detectors $[3,25]$. To be more specific, first, for the OMUD with ideal BS cooperation, the ASE can be evaluated from the formula [11]

$$
C_{\text {Asy }}=\frac{1}{\beta} \int_{0}^{\infty} \log _{2}\left(1+\frac{\lambda}{\sigma^{2}}\right) f_{\tilde{\boldsymbol{H}} \tilde{\boldsymbol{H}}^{H}}(\lambda) d \lambda,
$$


where $f_{\tilde{\boldsymbol{H}} \tilde{H}^{H}}(\lambda)$ is the asymptotic probability density function (PDF) of the eigenvalues of $\tilde{\boldsymbol{H}} \tilde{\boldsymbol{H}}^{H}$, here $\tilde{\boldsymbol{H}}$ is an $(N \times K)$ random matrix characterizing an equivalent channel model considered. With the aid of (29), analytical results for different scenarios have been derived in [11], some of which will be introduced for the OMUD with ideal BS cooperation in Section 4.1.

Second, for the other MUD schemes with/without BS cooperation, their ASE is obtained via deriving the asymptotic SINR attained by the MUDs, respectively, since we have [3]

$$
\begin{aligned}
C_{\text {Asy }} & =\lim _{K, N \rightarrow \infty} \frac{1}{K} \sum_{k=1}^{K} \log _{2}\left(1+\gamma_{k}\right) \\
& =\int_{0}^{1} \log _{2}(1+\gamma(x)) d x
\end{aligned}
$$

where $\gamma_{k}$ denotes the SINR of the $k$ th $(1 \leq k \leq K)$ user and $\gamma(x)$ is the corresponding asymptotic SINR with $x=$ $\frac{k}{K}$ uniformly distributed in $(0,1]$.

Let us now consider the ASE of the OMUD with ideal BS cooperation.

\subsection{Optimum multiuser detection with ideal BS cooperation}

In the multicell systems with the OMUD and ideal BS cooperation, the covariance matrix of (7) can correspondingly be expressed as

$$
\boldsymbol{V}=\frac{1}{3 N} \boldsymbol{P}=\frac{1}{3 N}\left[\begin{array}{lll}
3 \boldsymbol{J} & 3 \alpha^{2} \boldsymbol{J} & 3 \alpha^{2} \boldsymbol{J} \\
3 \alpha^{2} \boldsymbol{J} & 3 \boldsymbol{J} & 3 \alpha^{2} \boldsymbol{J} \\
3 \alpha^{2} \boldsymbol{J} & 3 \alpha^{2} \boldsymbol{J} & 3 \boldsymbol{J}
\end{array}\right]
$$

where $\boldsymbol{J}$ is an $(N \times K)$ matrix with elements of ones. It can be known from (31) that, $\boldsymbol{P}$ is a doubly-regular matrix ${ }^{\mathrm{a}}$ [11]. Based on this property, it can be shown [4] that the ASE of a circle Wyner model is equivalent to that of an isolate cell, in which the transmit power per user is increased to $\left(1+2 \alpha^{2}\right)$, owing to exploitation of the interference from its two adjacent cells. Therefore, the ASE of the OMUD with ideal BS cooperation can be readily obtained from that of the single-cell case with OMUD.

As shown in [11], the ASE of the single-cell setup is given by

$$
\begin{aligned}
C_{\text {Asy }}\left(\frac{1}{\sigma^{2}}, \beta\right)= & \log _{2}\left(1+\frac{1}{\sigma^{2}}-\frac{1}{4} \mathcal{F}\left(\frac{1}{\sigma^{2}}, \beta\right)\right) \\
& +\frac{1}{\beta} \log _{2}\left(1+\frac{\beta}{\sigma^{2}}-\frac{1}{4} \mathcal{F}\left(\frac{1}{\sigma^{2}}, \beta\right)\right) \\
& -\frac{\sigma^{2} \log _{2} e}{4 \beta} \mathcal{F}\left(\frac{1}{\sigma^{2}}, \beta\right),
\end{aligned}
$$

where $\mathcal{F}(x, y)=\left(\sqrt{x(1+\sqrt{y})^{2}+1}-\sqrt{x(1-\sqrt{y})^{2}+1}\right)^{2}$. Hence, for our considered model using the OMUD with ideal BS cooperation, the ASE can be obtained by replacing $1 / \sigma^{2}$ with $\left(1+2 \alpha^{2}\right) / \sigma^{2}[4]$ and is expressed as

$$
\begin{aligned}
C_{\text {Asy }}= & \log _{2}\left(1+\frac{u}{\sigma^{2}}-\frac{1}{4} \mathcal{F}\left(\frac{u}{\sigma^{2}}, \beta\right)\right) \\
& +\frac{1}{\beta} \log _{2}\left(1+\frac{u}{\sigma^{2}} \beta-\frac{1}{4} \mathcal{F}\left(\frac{u}{\sigma^{2}}, \beta\right)\right) \\
& -\frac{\sigma^{2} \log _{2} e}{4 u \beta} \mathcal{F}\left(\frac{u}{\sigma^{2}}, \beta\right)
\end{aligned}
$$

where $u=1+2 \alpha^{2}$.

As shown in [11], (32) is an increasing function of $1 / \sigma^{2}$. Thus, when replacing $1 / \sigma^{2}$ of the single-cell case by $(1+$ $\left.2 \alpha^{2}\right) / \sigma^{2}$ of the ideal cooperative multicell case, we are implied that the ASE of OMUD with ideal BS cooperation is higher than that of the OMUD for a corresponding isolate cell. The main reason behind is obvious, the OMUD with ideal BS cooperation is capable of exploiting intercell interference, and turning it into the useful signal, which provides diversity gain as well as power gain, and correspondingly increases the achievable spectral-efficiency. When considering the effect of the system load-factor $\beta, \mathcal{F}(x, \beta)$ is an increasing function of $\beta$. Then, we can deduce that (32) and (33) are decreasing functions of $\beta$, resulting in that the ASE of the OMUD for the singlecell setup and of the OMUD with ideal BS cooperation minishes, as the system load increases. More specifically, when $\beta \rightarrow \infty$, we have $\mathcal{F}(x, \beta)=4 x$. Explicitly, when applying it to (32) and (33), we can see that the ASE tends to zero, as $\beta \rightarrow \infty$.

\subsection{Optimum multiuser detection with ideal data exchange}

From Sections 3.2 and 3.4, we can see that the spectralefficiency of the OMUD with ideal data exchange, as shown in (10), shares the same form as that of the MMSESIC without BS cooperation shown in (21). The only difference between them is that a BS in the multicell systems employing the OMUD with ideal data exchange only conflicts interference from one adjacent cell, while a BS in the systems employing the MMSE-SIC without BS cooperation conflicts interference from its two adjacent cells. To obtain the ASE of (10) and (21), an intuitive approach is first to derive the asymptotic eigenvalue distribution of the matrices in the form of $\boldsymbol{H} \boldsymbol{H}^{H} \boldsymbol{\Sigma}^{-1}$. Then, the formula (29) in Section 4.1 is employed to obtain the ASE. However, the difficulty of this approach is to derive the asymptotic eigenvalue distribution of $\boldsymbol{H} \boldsymbol{H}^{H} \boldsymbol{\Sigma}^{-1}$. Fortunately, the ASE of the MMSE-SIC without BS cooperation can be analyzed by deriving its asymptotic SINR at each detection stage. Once the asymptotic SINR is obtained, the ASE can be evaluated with the aid of (30). Since (10) for the OMUD with ideal data exchange shares the same form of (21) for the MMSE-SIC without BS cooperation, the ASE 
of the OMUD with ideal data exchange can be directly obtained from the method adopted for deriving the ASE of the MMSE-SIC without BS cooperation, which will be detailed in Section 4.4.

In summary, with the aid of the analysis in Section 4.4, the ASE of the OMUD with ideal data exchange can be evaluated from (30) associated with $\gamma(x)$ determined by the equation

$$
\begin{aligned}
\gamma(x)= & \frac{(\beta x+1) \alpha^{2} \gamma^{2}(x)+\left(\beta x+\alpha^{2}+1\right) \gamma(x)+1}{\alpha^{2} \sigma^{2} \gamma^{2}(x)+\left(\alpha^{2} \sigma^{2}+\sigma^{2}+2 \alpha^{2} \beta\right) \gamma(x)+\sigma^{2}+\left(\alpha^{2}+1\right) \beta}, \\
& 0<x \leq 1
\end{aligned}
$$

It is worthy of noting that here the asymptotic SINR $\gamma(x)$ in (34) as well as the ASE expression of (30) are introduced only for the purpose of ASE evaluation, owing to the above argument that the spectral-efficiency of the OMUD with ideal data exchange and that of the MMSESIC without BS cooperation share the same form. However, we should realize that, in the OMUD with ideal data exchange, all the intracell users have the same asymptotic SINR. By contrast, under the MMSE-SIC without BS cooperation, the intracell users detected at different stages have different asymptotic SINR values.

\subsection{MMSE-MUD without BS cooperation}

The ASE of the MMSE-MUD and MMSE-SIC in presence of intercell interference has been studied in [3] for the direct-sequence code-division multiple-access (DSCDMA) systems over non-fading channels. It has been demonstrated that the elements of spreading sequences and the channel fading gains between transmit/receive antennas are equivalent for the purpose of asymptotic analysis [25]. Hence, in this contribution, we adopt the approaches provided in [3] to derive the asymptotic SINR in both this section and Section 4.4.

According to [3], when the MMSE-MUD without BS cooperation is considered, the multicell model, where each cell covers $K$ users, may be viewed as an equivalent single-cell system with $3 K$ users. Based on this equivalent single-cell model, the well-known Tse-Hanly Theorem [25] can then be used to obtain an implicit equation, which we can solve for an unique positive solution representing the asymptotic SINR of the MMSE-MUD. Specifically, in the multicell system considered in this article, as shown in Section 2, the equivalent single-cell model has one third of the users, each of which has a unit of received power due to the employment of ideal power control inside a cell, and two thirds of the users, each of which has the received power of $\alpha^{2}$. Hence, when specifically considering user 1 in Cell 1 , the interferers can be divided into two classes: $(K-1)$ intracell interferers with unit received power and $2 K$ intercell interferers with received power of $\alpha^{2}$. Consequently, applying ([25], Theorem 3.1), we can obtain

$$
\gamma=\frac{1}{\sigma^{2}+\beta\left(\frac{1}{1+\gamma}+\frac{2 \alpha^{2}}{1+\alpha^{2} \gamma}\right)}
$$

which, after some arrangements, can be written in the form

$$
\gamma \sigma^{2}+\beta\left[\frac{\gamma}{1+\gamma}+2 \frac{\alpha^{2} \gamma}{1+\alpha^{2} \gamma}\right]=1
$$

Furthermore, from (36), the SINR of user 1 in Cell 1 can be expressed as

$$
\gamma=\frac{\alpha^{2} \gamma^{2}+\left(\alpha^{2}+1\right) \gamma+1}{\alpha^{2} \sigma^{2} \gamma^{2}+\left(\alpha^{2} \sigma^{2}+\sigma^{2}+3 \alpha^{2} \beta\right) \gamma+\sigma^{2}+\left(2 \alpha^{2}+1\right) \beta}
$$

which is a fixed-point equation with a unique positive solution ([25], Proposition 3.2) that can be readily found through iterations.

In the considered multicell system, each user suffers from the interference generated by the other $(3 K-1)$ users in the same way as user 1 in Cell 1 . Hence, when $N, K \rightarrow \infty$, the asymptotic SINR is the same for any of the users. Thus, the ASE of the MMSE-MUD without BS cooperation is

$$
C_{\text {Asy }}=\log _{2}(1+\gamma)
$$

where $\gamma$ is the solution to (37).

Notice from (35)-(38) that $\gamma$ is independent of the uniform distributed variable $x$. This is because the SINR of all the users is the same, when the MMSE-MUD without BS cooperation is employed. Additionally, from (36), we can deduce that $\gamma$ is a decreasing function of $\beta$, yielding that $\gamma$ approaches zero as $\beta \rightarrow \infty$. In other words, we can have the similar conclusion that the ASE of MMSE-MUD without BS cooperation reduces, and finally tends to zero, as the system load increases, as that stated in Section 4.1.

\subsection{MMSE-SIC without BS cooperation}

In comparison with the MMSE-MUD without BS cooperation, as analyzed in Section 4.3, when the MMSE-SIC without BS cooperation is considered, the SINR of different users may be different, depending on the detection orders. As seen in (15), user $k$ only suffers interference from the $(K-k)$ users detected after user $k$. Therefore, similar to (36) and remembering that there are $(K-k)$ intracell interfering users, the implicit equation determining the asymptotic SINR is

$$
\gamma(x) \sigma^{2}+\beta\left[(1-x) \frac{\gamma(x)}{1+\gamma(x)}+2 \frac{\alpha^{2} \gamma(x)}{1+\alpha^{2} \gamma(x)}\right]=1
$$


Note that, we have (39) because, at a given detection stage, the asymptotic SINR at different BSs is the same, owing to the symmetric characteristic of our multicell system model.

Then, after some arrangements, the asymptotic SINR of the $k$ th $(1 \leq k \leq K, K \rightarrow \infty)$ user can be found via the equation

$$
\gamma(x)=\frac{(\beta x+1) \alpha^{2} \gamma^{2}(x)+\left(\beta x+\alpha^{2}+1\right) \gamma(x)+1}{\alpha^{2} \sigma^{2} \gamma^{2}(x)+\left(\alpha^{2} \sigma^{2}+\sigma^{2}+3 \alpha^{2} \beta\right) \gamma(x)+\sigma^{2}+\left(2 \alpha^{2}+1\right) \beta}
$$

by the iteration approach. Note that, in the above equation, $x$ is uniformly distributed in $(0,1]$. Finally, the ASE of the multicell systems employing the MMSE-SIC without BS cooperation can be evaluated from (30) upon substituting (40). From (39) we can find that, when $x$ is given, $\gamma(x)$ is a decreasing function with $\beta$. Hence, it tends to zero, as $\beta \rightarrow \infty$. However, when comparing (39) with (36), we can learn that the decreasing rate of $\gamma(x)$ in (39) is slower than that of $\gamma(x)$ in (36) due to the fraction of $(1-x)$ seen in (39). From this we are implied that the corresponding ASE decreases slower than that of the MMSE-MUD, as the system load-factor $\beta$ increases.

Note furthermore that, in the context of the multicell system employing the OMUD-PIC with data exchange, as discussed in Section 4.2, (10) implies that its ASE is the same as that achieved by the MMSE-SIC experiencing intercell interference from only one cell. Hence, similar to (39), the asymptotic SINR satisfies the implicit equation

$$
\gamma(x) \sigma^{2}+\beta\left[(1-x) \frac{\gamma(x)}{1+\gamma(x)}+\frac{\alpha^{2} \gamma(x)}{1+\alpha^{2} \gamma(x)}\right]=1,
$$

from which (34) can be obtained.

It is worthy of noting again that, when the OMUD-PIC with data exchange is employed, all the individual users in a cell achieve the same rate. By contrast, when the MMSESIC without BS cooperation is employed, different users in a cell may communicate with different rates.

\subsection{MMSE-SIC with ideal data exchange}

As analyzed in Section 3.5, when the MMSE-SIC is implemented with ideal data sharing among BSs, the detection of user $k$ of a cell conflicts interference from $(K-k)$ intracell users and $2(K-k+1)$ intercell users with $(K-k+1)$ users from each of the two adjacent cells. Therefore, following the analysis in Section 4.4, we can obtain the implicit equation

$$
\gamma(x) \sigma^{2}+\beta(1-x)\left[\frac{\gamma(x)}{1+\gamma(x)}+2 \frac{\alpha^{2} \gamma(x)}{1+\alpha^{2} \gamma(x)}\right]=1
$$

Note that, the above equation is verified, as $(K-$ $k+1) / N=(K-k) / N=\beta(1-x)$, where $x=k / K$, when $N, K \rightarrow \infty$. Consequently, after rearranging the above equation, we can obtain a fixed-point equation

$$
\begin{aligned}
\gamma(x)= & \frac{\alpha^{2} \gamma^{2}(x)+\left(\alpha^{2}+1\right) \gamma(x)+1}{\alpha^{2} \sigma^{2} \gamma^{2}(x)+\left[\alpha^{2} \sigma^{2}+\sigma^{2}+3 \alpha^{2} \beta(1-x)\right] \gamma(x)} \\
& +\sigma^{2}+\left(2 \alpha^{2}+1\right) \beta(1-x)
\end{aligned}
$$

which is suitable for using iterative approach to obtain the unique positive solution $\gamma(x)$, as mentioned in the previous sections.

Additionally, similar to the analysis in previous sections, for a given $x, \gamma(x)$ is a decreasing function of $\beta$ and tends to zero, as $\beta \rightarrow \infty$. The difference here is the extra factor of $(1-x)$ with regard to intercell users, which is resulted from the intercell interference cancellation via data exchange among BSs. Consequently, the ASE decreasing rate of the MMSE-SIC with ideal data exchange is even lower than that of the MMSE-SIC without BS cooperation, as analyzed in Section 4.4.

So far, we have obtained the formulas for estimating the ASE of all the schemes considered in this contribution. We found that the ASE of all the schemes tends to zero, as $\beta \rightarrow \infty$, but the decreasing rates may be different. In the next section, we try to gain some insights from the cases when $\beta \rightarrow 0$, which corresponds to the situations of massive MIMO systems [15,17].

\section{Asymptotic spectral-efficiency when $\beta \rightarrow 0$}

In this section, we analyze the asymptotic spectralefficiency of the schemes considered in the previous two sections, when the system load-factor $\beta$ tends to zero. This corresponds to the lightly loaded multicell systems, where each BS supports a relatively low number of users, but has a large number of degrees of freedom provided, such as, by a large BS antenna array. Let us first consider the single-cell scenario. According to (32), the asymptotic spectral-efficiency of the single-cell system can be expressed in terms of $\beta$ as

$$
\begin{aligned}
C_{\text {Asy }}(\beta)= & \log _{2}\left(1+\frac{1}{\sigma^{2}}\right) \\
& +\frac{1}{\beta} \log _{2}\left(1+\frac{\beta}{\sigma^{2}}-\frac{1}{4} \mathcal{F}\left(\frac{1}{\sigma^{2}}, \beta\right)\right) \\
& -\frac{\sigma^{2} \log _{2} e}{4 \beta} \mathcal{F}\left(\frac{1}{\sigma^{2}}, \beta\right)
\end{aligned}
$$


For convenience, let $\gamma=1 / \sigma^{2}$. Then, when $\beta$ tends to zero, we can apply the L'Hopital rule [26] to obtain

$$
\begin{aligned}
\lim _{\beta \rightarrow 0} C_{\text {Asy }}(\beta)= & \log _{2}(1+\gamma)+\frac{\log _{2} e}{1+\beta \gamma-\frac{1}{4} \mathcal{F}(\gamma, \beta)} \\
& \times\left(\gamma-\frac{1}{4} \mathcal{F}^{\prime}(\gamma, \beta)\right)-\frac{\log _{2} e}{4 \gamma} \mathcal{F}^{\prime}(\gamma, \beta) \\
= & \log _{2}(1+\gamma) \\
& +\log _{2} e\left(\gamma-\frac{1}{4} \mathcal{F}^{\prime}(\gamma, \beta)-\frac{1}{4 \gamma} \mathcal{F}^{\prime}(\gamma, \beta)\right)
\end{aligned}
$$

where $\mathcal{F}^{\prime}(\gamma, \beta)=\lim _{\beta \rightarrow 0^{+}} \frac{d \mathcal{F}(\gamma, \beta)}{d \beta}$. Upon carrying out the differentiation of $\mathcal{F}(\gamma, \beta)$ with respect to $\beta$ and after some arrangements, we obtain

$$
\mathcal{F}^{\prime}(\gamma, \beta)=\lim _{\beta \rightarrow 0^{+}} \mathcal{F}^{\frac{1}{2}}(\gamma, \beta) \beta^{-\frac{1}{2}} \frac{2 \gamma}{\sqrt{\gamma+1}} .
$$

Let, in the above equation, $\beta^{-\frac{1}{2}}=t$. Then, we can apply again the L'Hopital rule to derive

$$
\mathcal{F}^{\prime}(\gamma, \beta)=\frac{4 \gamma^{2}}{\gamma+1}
$$

Finally, substituting this result and $\gamma=1 / \sigma^{2}$ into (45) yields

$$
\lim _{\beta \rightarrow 0} C_{\text {Asy }}(\beta)=\log _{2}\left(1+\frac{1}{\sigma^{2}}\right)
$$

which is the spectral-efficiency of a single-cell MIMO system, when $\beta \rightarrow 0$.

When the OMUD with ideal BS cooperation is considered, as analyzed in Section 4.2, the ASE is expressed as (33) and is equal to that of a separate cell with each user transmitting signals using the power of $\left(1+2 \alpha^{2}\right)$. Hence, by replacing $1 / \sigma^{2}$ with $\left(1+2 \alpha^{2}\right) / \sigma^{2}$ in (48), the ASE achieved by the OMUD with ideal BS cooperation for the multicell systems with $\beta \rightarrow 0$ can be expressed as

$$
\lim _{\beta \rightarrow 0} C_{\text {Asy }}(\beta)=\log _{2}\left(1+\frac{1}{\sigma^{2}}+\frac{2 \alpha^{2}}{\sigma^{2}}\right)
$$

In the context of the other schemes, namely, the MMSEMUD and MMSE-SIC without BS cooperation, and the OMUD and MMSE-SIC with ideal data exchange, when $\beta$ tends to zero, we can readily find from (36), (39), (41), and (42) that their asymptotic SINR is the same and is given by $\gamma=1 / \sigma^{2}$. Hence, when $\beta$ tends to zero, their ASE can be expressed as (48).

From the above analysis, we are implied that, when a multicell system is lightly loaded, yielding $\beta \rightarrow 0$, the schemes of MMSE-MUD and MMSE-SIC without BS cooperation, and the OMUD and MMSE-SIC with ideal data exchange achieve the same spectral-efficiency, which is equal to the single-cell bound. By contrast, as shown in (49), the spectral-efficiency attainable by the OMUD with ideal BS cooperation may be higher than the single-cell bound. Furthermore, higher intercell interference generates higher spectral-efficiency for the OMUD with ideal BS cooperation. Hence, in a lightly loaded multicell system, either no BS cooperation is necessary or ideal BS cooperation has to be implemented in order to achieve an improved spectral-efficiency. However, as discussed previously in Section 3.1, implementing ideal BS cooperation requires exchange of both CSI and observations among BSs, which demands extremely high complexity.

Therefore, at least for the near future cellular communications, massive MIMO [15,17] might be one of reasonable candidates. In massive MIMO systems, the number of users supported per cell is supposed to be significantly lower than the number of antenna elements per BS, which may be on the order of hundreds. Hence, they are typical systems of lightly loaded. Therefore, in this type of massive MIMO systems, no BS cooperation is necessary, as ideal BS cooperation is seems impossible due to the extreme requirement of backhaul resources. Furthermore, as the analysis in $[15,17]$ shows, in massive MIMO systems, lowcomplexity single-user detection, such as matched-filter (MF) based detection, tends to optimum and no MUD is necessary. This also makes the system design easier.

In the following section, we provide a range of results to demonstrate and compare the capacity or spectralefficiency achievable by the multicell systems, when the various MUD and/or BS cooperation schemes are considered.

\section{Performance results}

In this section, performance results, which were either obtained from simulation or evaluated from the formulas derived, will be provided, in order to compare the achievable spectral-efficiency of the multicell MIMO systems employing different MUD and BS cooperation schemes considered in Section 2. The spectral-efficiency is expressed in terms of bits/s/Hz/User, representing the number of bits per second per Hertz per user. As a benchmark, in these figures, the spectral-efficiency of a single isolate cell is also included. Specifically, in the not so large system, the impacts of the system load, which is explained by the number of users per cell, SNR and the intercell interference strength, which is reflected by the parameter $\alpha$, are demonstrated. From the figures, we can obtain the implication about the consistency between the results obtained by simulation and asymptotic analysis. Furthermore, as a benchmark for comparison, the spectral-efficiency of the zero-forcing MUD without BS cooperation [20,27], which is obtained by simulations, is also included in some figures. For the multicell systems, where every BS employs a large number of antennas or supports a large number of users, it is extremely hard to obtain results via simulation. In these case, we will only 
provide the results evaluated from the formulas derived by asymptotic analysis in Section 4. Additionally, the keys used in the figures are summarized for convenience as follows:

- Ideal Cooperation: OMUD with ideal BS cooperation considered in Sections 3.1 and 4.1;

- Single-Cell Bound: Spectral-efficiency of a single isolate cell;

- OMUD-PIC-DE: OMUD-PIC supported by ideal data exchange considered in Sections 3.2 and 4.2;

- MMSE-MUD: MMSE-MUD without BS cooperation considered in Sections 3.3 and 4.3;

- MMSE-SIC: MMSE-SIC without BS cooperation considered Sections 3.4 and 4.4;

- MMSE-SIC-DE: MMSE-SIC with ideal data exchange considered in Sections 3.5 and 4.5.

- ZF-MUD: Zero-forcing MUD without BS cooperation $[20,27]$.

Figure 3 shows the effect of the number of users per cell on the spectral-efficiency of multicell systems, when $\alpha=0.5, N=4$ and SNR $=10 \mathrm{~dB}$. First, when comparing the results obtained by simulation and that evaluated from the formulas obtained by asymptotic analysis, we can see that, except the MMSE-SIC-DE scheme, they agree with each other very well, even when the number of users per cell is very low, such as $K=2$, 3, etc. For the MMSE-SIC-DE scheme, the simulated result does not match closely with the ASE, when the number of users per cell is small. This is because, for the ASE, the assumption of $(K-k+1) / N=(K-k) / N$ is used, as shown in Section 4.5. Explicitly, when $K$ is small, this assumption is not very accurate, which causes the diversity between

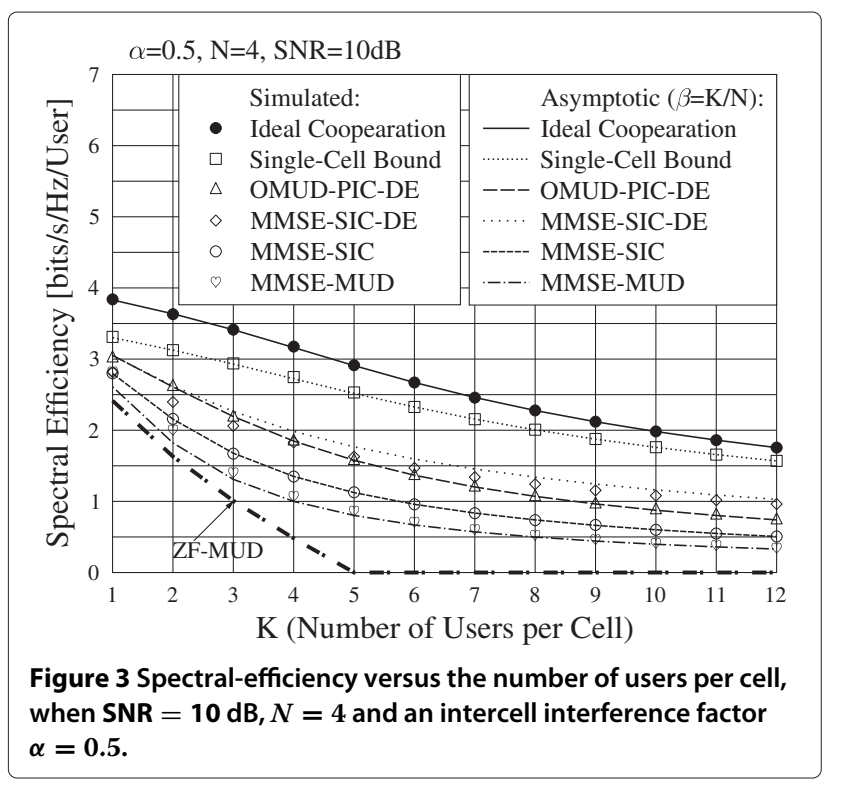

the simulated spectral-efficiency and the ASE. By contrast, as $K$ increases, we can observe that the ASE evaluated from formulas becomes very close to the simulated results. From results of Figure 3, we can observe that the spectral-efficiency per user decreases, as the number of users per cell increases, due to the increased interference. Among the detection and cooperation schemes considered, the scheme of OMUD with ideal BS cooperation achieves the highest spectral-efficiency, which is even higher than the single-cell bound, owing to its capability to exploit intercell interference. By contrast, for all the other schemes, intercell interference is a deterioration factor. Correspondingly, as seen in Figure 3, their spectral-efficiency is lower than the single-cell bound.

When comparing the two detection schemes with ideal data exchange, the spectral-efficiency of the OMUD-PICDE scheme is slightly higher than that of the MMSE-SICDE scheme, when the system load is low. However, as the system load increases, their spectral-efficiency has a cross and, after it, the MMSE-SIC-DE scheme is capable of achieving higher spectral-efficiency than the OMUDPIC-DE scheme. The reason behind the above observation is that, when the number of user per cell is relatively low, intracell interference dominates the detection performance. As the OMUD provides more reliable detection than the MMSE-MUD, the OMUD-PIC-DE scheme yields higher spectral-efficiency than the MMSE-SIC-DE scheme. However, when the number of users per cell increases, intercell interference becomes domination of the achievable spectral-efficiency. According to our analysis in Section 3.2, the OMUD-PIC-DE scheme can only suppress the interference from one of the two adjacent cells. By contrast, as shown in Section 3.5, the MMSESIC-DE scheme is capable of suppressing the interference from both adjacent cells. Consequently, the MMSE-SICDE scheme outperforms the OMUD-PIC-DE scheme.

Finally, when comparing the MMSE-MUD, MMSE-SIC, and the ZF-MUD, all of which do not carry out BS cooperation, the spectral-efficiency achieved by the MMSE-SIC is always higher than that attainable by the MMSE-MUD or ZF-MUD, provided that the number of users per cell is more than one. Among the three, the ZF-MUD scheme is always the worst, whose achievable spectral-efficiency is zero when the system is overloaded. This implies that the ZF-MUD scheme is incapable of providing the reliable communication in an overloaded system.

Figure 4 shows the spectral-efficiency versus SNR performance for a multicell system with an intercell interference factor $\alpha=0.5$. In our simulations, we assumed that $N=K=4$, yielding $\beta=1$. Correspondingly, in the numerically evaluated results, we used $\beta=1$. As shown in Figure 4 as well as in Figure 3, explicitly, intercell interference has a strong impact on the achievable spectralefficiency. As the OMUD with ideal BS cooperation can 


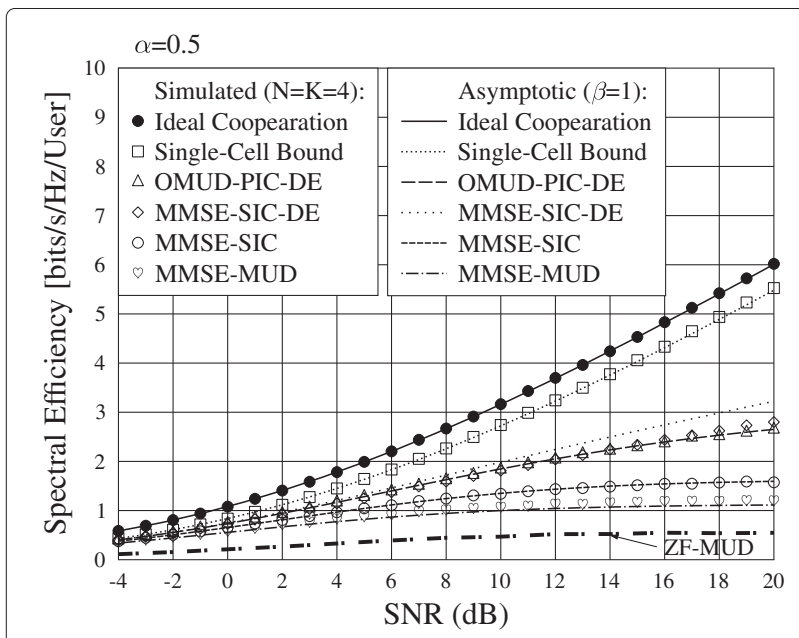

Figure 4 Spectral-efficiency versus SNR for a multicell system with the parameters $N=K=4$ and $\alpha=0.5$. make use of intercell interference, its spectral-efficiency is always higher than that of the other schemes that are unable to use interference. However, when without using inter-BS data exchange, as shown by the curves corresponding to the MMSE-MUD, MMSE-SIC and the ZF-MUD, the achieved spectral-efficiency finally becomes interference-limited and does not increases, when the SNR increases beyond a certain value. By contrast, when inter-BS data exchange is available, as shown by the curves corresponding to the OMUD-PIC-DE and MMSE-SIC$\mathrm{DE}$, the spectral-efficiency always increases with the SNR, although their achieved spectral-efficiency is lower than that of the single-cell bound. From Figure 4, we can also observe that the spectral-efficiency evaluated from the asymptotic formulas agrees well with that obtained via simulation. The only exception is the MMSE-SIC-DE scheme. When the SNR is high, we can see that the asymptotic results may over estimate the spectral-efficiency achievable by the MMSE-SIC-DE scheme. Again, as stated associated with Figure 3, this is because the assumption that $(K-k+1) / N=(K-k) / N$, when $N, K \rightarrow \infty$, is used in the asymptotic analysis. Specifically, in Figure 4, we used $N=K=4$, which makes the assumption not accurate. Consequently, the difference between simulated and asymptotic results is relatively large, especially when SNR is high. To support our argument, in Figure 5 we assumed that $N=K=16$. Explicitly, the simulated results agree well with the corresponding asymptotic results even when the SNR is relatively high. Thus, we can be confident that the spectral-efficiency of a multicell system can in general be predicted by the formulas derived from asymptotic analysis in this article.

The impact of intercell interference factor $\alpha$ on the spectral-efficiency is illustrated in Figure 6 for $N=K=$

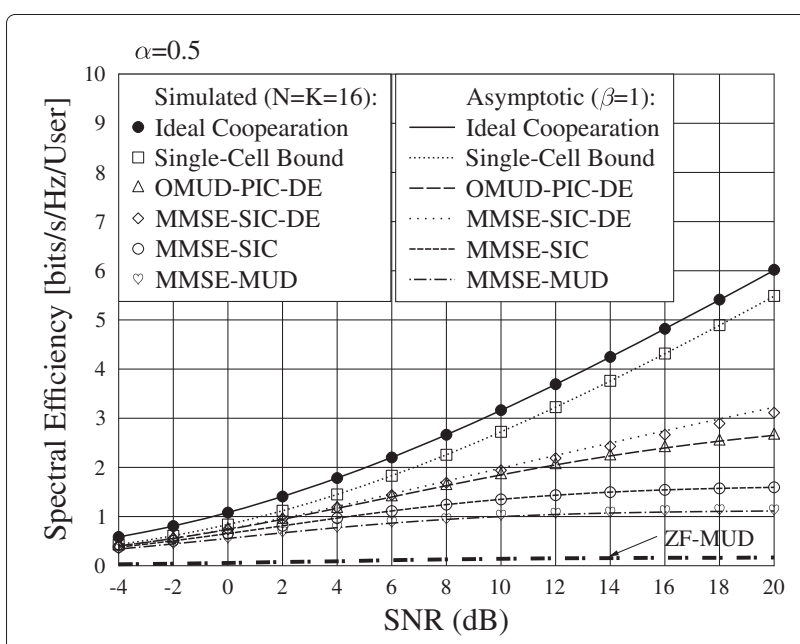

Figure 5 Spectral-efficiency versus SNR for a multicell system with the parameters $N=K=16$ and $\alpha=0.5$.

4 and Figure 7 for $N=K=8$, respectively, when $\mathrm{SNR}=10 \mathrm{~dB}$. When $\alpha=0$ meaning no intercell interference, except the MMSE-MUD and ZF-MUD, all the schemes achieve the same spectral-efficiency that is equal to the single-cell bound. As intercell interference, i.e., the value of $\alpha$, increases, the spectral-efficiency of the OMUD with ideal BS cooperation increases, owing to the increased information acquired from the neighboring BSs. Since all the other schemes considered are unable to make use of intercell interference, correspondingly, their achieved spectral-efficiency decreases, when the value of $\alpha$ becomes larger. However, the two schemes with data exchange among BSs significantly outperform the other schemes without BS cooperation.

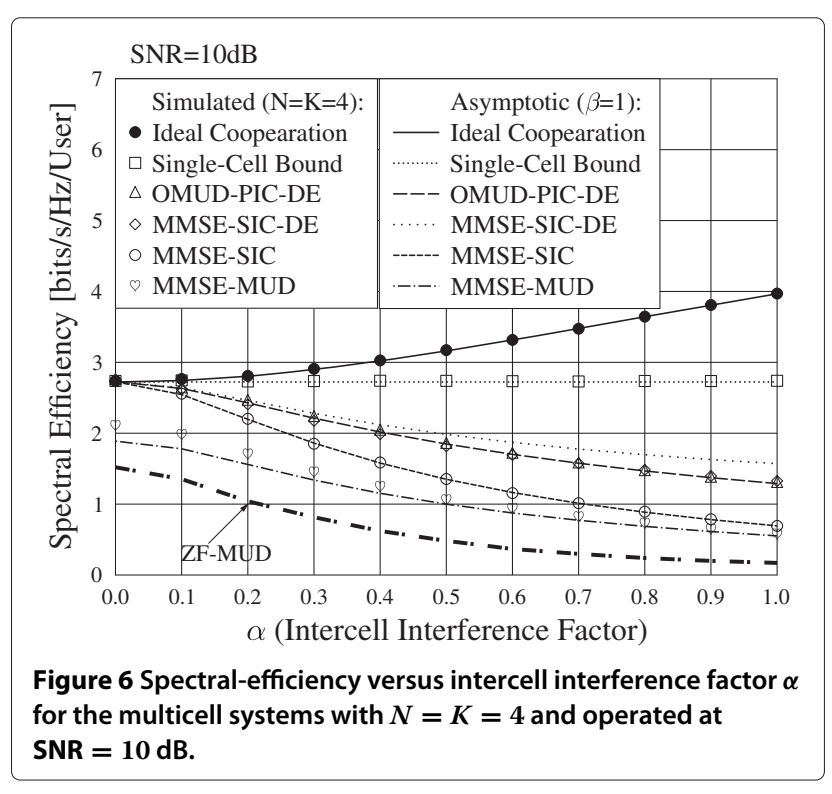




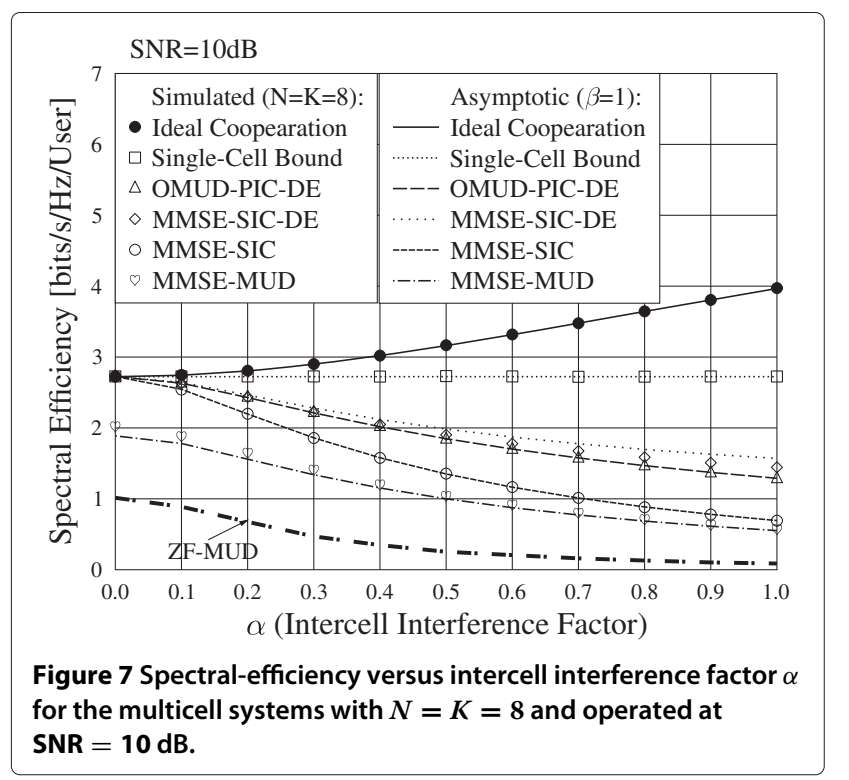

As in Figures 3, 4, and 5, the results in Figures 6 and 7 illustrate that the asymptotic results in general agree well with the results obtained via simulation. This observation becomes more declared, when $N=K=8$ (Figure 7) is considered. As shown in Figure 6 corresponding to $N=K=4$, for both the MMSE-MUD and the MMSESIC-DE schemes, the asymptotic spectral-efficiency has certain difference from that obtained by simulation. However, when $N=K=8$, as shown in Figure 7, this difference becomes smaller.

Figure 8 shows the asymptotic spectral-efficiency of multicell systems versus the system load-factor $\beta$, when various MUD and BS cooperation schemes are considered. First, it can be shown that, when $\beta \rightarrow 0$, except

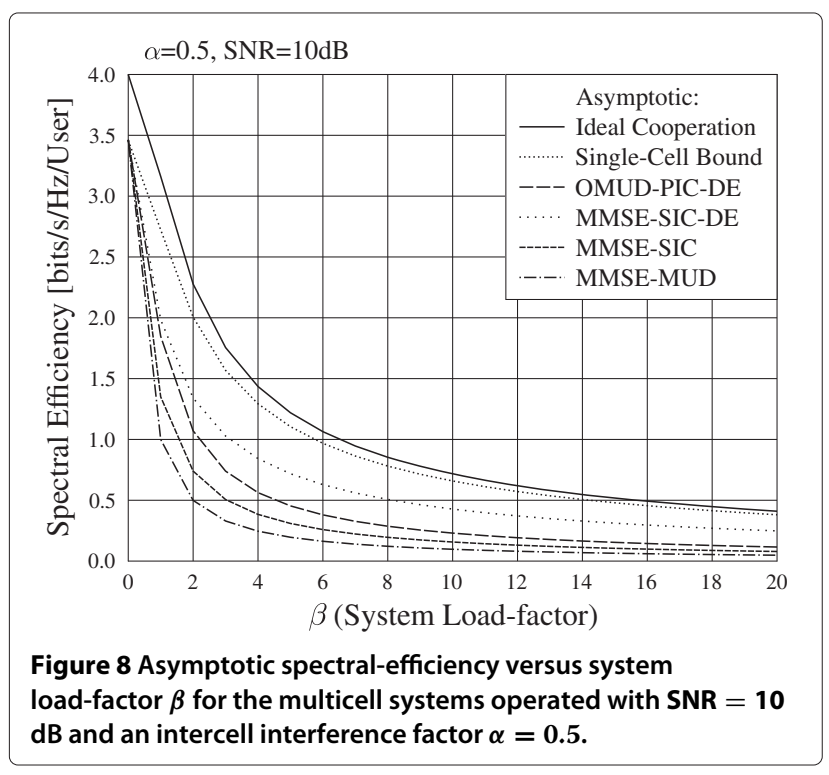

the OMUD with ideal BS cooperation, all the other schemes considered achieve the same spectral-efficiency limit of $\log _{2} 11 \mathrm{bits} / \mathrm{s} / \mathrm{Hz} /$ User, regardless of using or without using BS cooperation. This observation verifies our analysis in Section 5. Therefore, when each BS of a multicell system employs a large antenna array to support a relatively low number of users, exchanging data among BSs does not result in any spectral-efficiency benefit. In this case, each BS can separately detect its users, which is sufficient to attain all the spectral-efficiency that is achievable with the aid of data exchange among BSs. By contrast, when the ideal BS cooperation is implemented, which requires exchange of both data and CSI, an increased spectral-efficiency can be obtained, as shown in Figure 8. According to our detailed analysis in Section 5, we can find that, for the parameters used in Figure 8, the spectral-efficiency achieved by the OMUD with ideal BS cooperation is $4 \mathrm{bits} / \mathrm{s} / \mathrm{Hz} /$ User, which is higher than $\log _{2} 11 \mathrm{bits} / \mathrm{s} / \mathrm{Hz} /$ User.

Second, from the results of Figure 8, we can find that, as $\beta$ increases, the asymptotic spectral-efficiency of all the schemes considered decreases, but at different rates, as described in Section 4. Specially, the ASE of the OMUD with ideal BS cooperation gradually converges to the single-cell bound. Therefore, when a multicell system is heavily loaded, i.e., when the value of $\beta$ is high, the maximum spectral-efficiency achievable is equivalent to that achieved by a system, whose cells are operated separately without intercell interference. Among the other schemes, as seen in Figure 8, as the value of $\beta$ increases, the MMSESIC-DE stands out from the others and achieves the highest spectral-efficiency, which is even better than that of the OMUD-PIC-DE. Considering the fact that the MMSE-SIC has much lower complexity than the OMUD-PIC [23], the

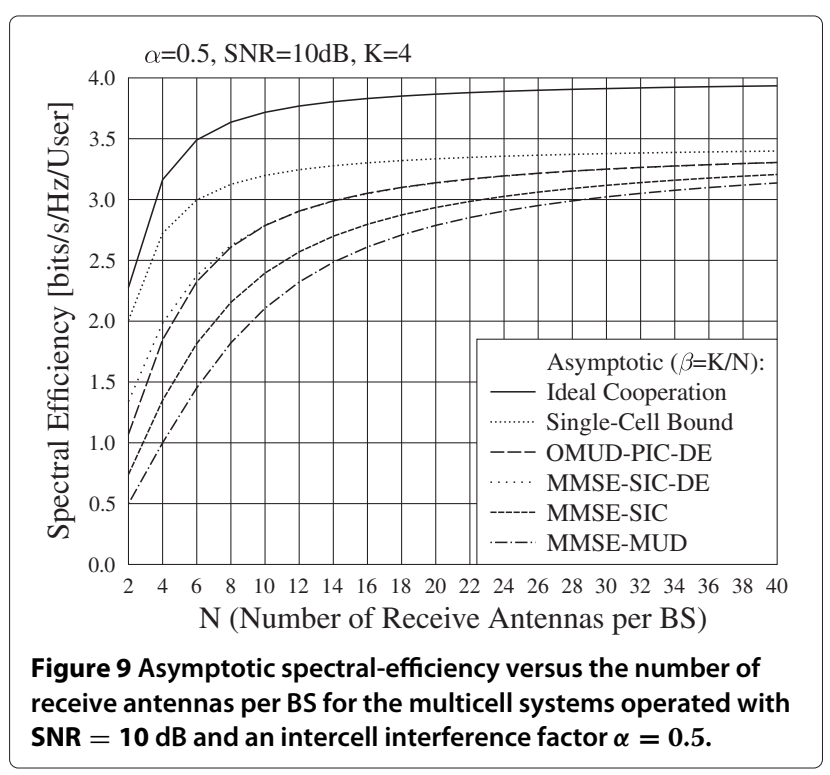


MMSE-SIC with data exchange assisted BS cooperation may constitute a promising MCP scheme that is suitable for implementation in practical multicell systems.

In order to illustrate the effect of system load on the spectral-efficiency, in Figure 9, we depict the spectralefficiency against the number of receive antennas per BS for the various MUD and BS cooperation schemes considered. As shown in Figure 9, the number of users per cell, $K$, is fixed to 4 , while the number of receive antennas per BS, $N$, varies from 2 to 40. As seen in Figure 9, the spectral-efficiency attained by the OMUD with ideal BS cooperation is always the highest. By contrast, as the number of receive antennas per BS increases, the spectralefficiency achieved by the other schemes converges to the single-cell bound.

\section{Conclusions}

In this contribution, we have investigated the spectralefficiency of uplink multicell MIMO systems by both simulation and asymptotic analysis, when different BS operational schemes and MUD schemes are invoked. The impacts of system load, SNR and intercell interference strength on the achievable spectral-efficiency have been studied and demonstrated. Our studies and performance results demonstrate that the asymptotic results usually agree well with the simulated results, provided that the number of users per cell or/and the number of receive antennas per BS are not too low. Generally, in multicell MIMO systems, employing BS cooperation supported by data or/and CSI exchange among different BSs is beneficial to improving their spectral-efficiency. Owing to its capability to exploit intercell interference, the scheme of OMUD with ideal BS cooperation outperforms all the other schemes, with regard to their achievable spectral-efficiency. However, implementing the OMUD with ideal BS cooperation demands extremely high complexity and backhaul resources, which are hard to provide in practice. The scheme of MMSE-SIC with data exchange only is capable of achieving significantly higher spectralefficiency than the schemes without BS cooperation. As the MMSE-SIC is a low-complexity MUD and the BS cooperation only requires data exchange, the MMSE-SIC supported by data exchange among BSs may constitute a promising MCP scheme that is suitable for implementation in practical multicell systems.

Furthermore, our studies demonstrate that, in a lightly loaded multicell system, BS cooperation does not yield much improvement of spectral-efficiency. By contrast, in a heavily loaded multicell system, the maximum spectralefficiency achieved by using OMUD with ideal BS cooperation converges to the spectral-efficiency achieved by a multicell system, where the invoked cells are operated separately without intercell interference. Additionally, in a heavily loaded multicell system, the MMSE-SIC with data exchange achieves a higher spectral-efficiency than the OMUD-PIC supported by data exchange.

\section{Endnote}

${ }^{\text {a An }} N \times K$ matrix is asymptotic doubly-regular, if $\lim _{K \rightarrow \infty} \frac{1}{K} \sum_{j=1}^{K} 1\left\{P_{i, j} \leq \alpha\right\}$ and $\lim _{N \rightarrow \infty} \frac{1}{N} \sum_{i=1}^{N} 1\left\{P_{i, j} \leq \alpha\right\}$ are independent of $i$ and $j$ for all $\alpha \in \mathbb{R}$, when the ratio $\beta=K / N$ converges to a constant.

\section{Competing interests \\ The authors declare that they have no competing interests.}

\section{Acknowledgements}

This study was supported in part by the National Basic Research Program of China (973 Program, Grant No. 2010CB731803) and the National Natural Science Foundation of China (Grant Nos. 60921001 and 61071072). The financial support of the China Scholarship Council (CSC) is also greatly acknowledged.

\section{Author details}

${ }^{1}$ School of Electronic and Information Engineering, Beihang University, Beijing 100191, China. ${ }^{2}$ School of Electronics and Computer Science, University of Southampton, Southampton, SO17 1BJ, UK.

Received: 11 July 2012 Accepted: 5 February 2013

Published: 15 March 2013

\section{References}

1. A Wyner, Shannon-theoretic approach to a Gaussian cellular multiple-access channel. IEEE Trans. Inf. Theory. 40(6), 1713-1727 (1994)

2. D Gesbert, S Hanly, H Huang, SS Shitz, O Simeone, W Yu, Multi-cell MIMO cooperative networks: a new look at interference. IEEE J. Sel. Areas Commun. 28(9), 1380-1408 (2010)

3. B Zaidel, S Shamai, S Verdú, Multicell uplink spectral efficiency of coded DS-CDMA with random signatures. IEEE J. Sel. Areas Commun. 19(8), 1556-1569 (2001)

4. O Somekh, B Zaidel, S Shamai, Spectral efficiency of joint multiple cell-site processors for randomly spread DS-CDMA systems. IEEE Trans. Inf. Theory. 53(7), 2625-2637 (2007)

5. O Somekh, B Zaidel, S Shamai, Sum rate characterization of joint multiple cell-site processing. IEEE Trans. Inf. Theory. 53(12), 4473-4497 (2007)

6. M Grieger, P Marsch, G Fettweis, J Cioffi, in IEEE Global Telecommunications Conference, GLOBECOM. On the performance of compressed interference forwarding for uplink base station cooperation, (Honolulu, Hawaii, USA, 2009), pp. 1-6

7. P Marsch, G Fettweis, Uplink CoMP under a constrained backhaul and imperfect channel knowledge. IEEE Trans. Wirel. Commun. 10(6), 1730-1742 (2011)

8. H Dai, H Poor, Asymptotic spectral efficiency of multicell MIMO systems with frequency-flat fading. IEEE Trans. Signal Process. 51(11), 2976-2988 (2003)

9. J Lee, D Toumpakaris, W Yu, Interference mitigation via joint detection. IEEE J. Sel. Areas Commun. 29(6), 1172-1184 (2011)

10. A Tulino, S Verdú, A Lozano, in Proceedings. 2003 IEEE Information Theory Workshop (ITW'03). Capacity of antenna arrays with space, polarization and pattern diversity, (Paris, France, 2003), pp. 324-327

11. A Tulino, S Verdú, Random Matrix Theory and Wireless Communications. (Now Publisher, Amsterdam, The Netherlands, 2004)

12. W Hachem, P Loubaton, J Najim, Determinstic equivalents for certain functionals of large random matrices. Annals Appl. Probab. 17(3), 875-930 (2007)

13. R Speicher, Combinatorial Theory of The Free Product With Amalgamation And Operator-valued Free Probability Theory. (American Mathematical Society, Providence, Rhode Island, 1998)

14. RSA Nica, Lectures on the combinatorics of free probability. (Cambridge University Press, Cambridge, 2006)

15. T Marzetta, Noncooperative cellular wireless with unlimited numbers of base station antennas. IEEE Trans. Wirel. Commun. 9(11), 3590-3600 (2010) 
16. J Hoydis, S ten Brink, M Debbah, in IEEE, The 49th Annual Allerton Conference on Communication, Control, and Computing (Allerton). Massive MIMO: how many antennas do we need?, (Monticello, Illinois, USA, 2011), pp. 545-550

17. F Rusek, D Persson, L Buon Kiong, E Larsson, T Marzetta, O Edfors, F Tufvesson, Scaling up MIMO: opportunities and challenges with very large arrays. IEEE Signal Process. Mag. 30(1), 40-60 (2013)

18. R Blum, MIMO capacity with interference. IEEE J. Sel. Areas Commun. 21(5), 793-801 (2003)

19. E Telatar, Capacity of multi-antenna gaussian channels. Europ. Trans. Telecommun. 10(6), 585-595 (1999)

20. S Verdú, Multiuser Detection. (Cambridge University Press, Cambridge, 1998)

21. L-L Yang, Multicarrier Communications. (John Wiley, Chichester, 2009)

22. S Shamai, S Verdú, The impact of frequency-flat fading on the spectral efficiency of CDMA. IEEE Trans. Inf. Theory. 47(4), 1302-1327 (2001)

23. L-L Yang, Receiver multiuser diversity aided multi-stage minimum mean-square error detection for heavily loaded DS-CDMA and SDMA systems. IEEE Trans. Commun. 58(12), 3397-3404 (2010)

24. D Tse, P Viswanath, Fundermentals of Wireless Communication. (Cambridge University Press, Cambridge, 2005)

25. D Tse, S Hanly, Linear multiuser receivers: effective interference, effective bandwidth and user capacity. IEEE Trans. Inf. Theory. 45(2), 641-657 (1999)

26. R Adams, C Essex, Calculus: a Complete Course, 7th edn. (Prentice Hall, Canada, 2010)

27. S-E Elayoubi, T Chahed, G Hebuterne, in IEEE Global Telecommunications Conference, GLOBECOM, vol. 1. On the capacity of multi-cell UMTS, (San Francisco, USA, 2003), pp. 487-491

Cite this article as: Ju et al:: Comparative study of various multiuser detection and base-station cooperation schemes for uplink multicell systems. EURASIP Journal on Wireless Communications and Networking 2013 2013:71.

\section{Submit your manuscript to a SpringerOpen ${ }^{\mathcal{O}}$ journal and benefit from:}

- Convenient online submission

- Rigorous peer review

- Immediate publication on acceptance

- Open access: articles freely available online

- High visibility within the field

- Retaining the copyright to your article

Submit your next manuscript at $\boldsymbol{\wedge}$ springeropen.com 